\title{
Labyrinthe
}

38 | 2012 (1)

L'éloquence des singes

\section{À propos de l'homme, ou comment repenser la sélection naturelle du langage humain}

\author{
Terrence W. Deacon
}

Traducteur : Marc Aymes

\section{OpenEdition \\ Journals}

Édition électronique

URL : http://journals.openedition.org/labyrinthe/4242

DOI : $10.4000 /$ labyrinthe.4242

ISSN : 1950-6031

Éditeur

Hermann

Édition imprimée

Date de publication : 1 avril 2012

Pagination : 27-37

ISBN : 9782705682361

Référence électronique

Terrence W. Deacon, «À propos de l'homme, ou comment repenser la sélection naturelle du langage humain », Labyrinthe [En ligne], 38 | 2012 (1), mis en ligne le 01 avril 2014, consulté le 19 avril 2019. URL : http://journals.openedition.org/labyrinthe/4242 ; DOI : 10.4000/labyrinthe.4242 


\title{
À propos de l'homme, ou comment repenser la sélection naturelle du langage humain
}

\author{
Terrence W. DEACON
}

Le loufoque Garner rêvait, avec d'autres, de singes domestiqués, s'approchant encore du niveau des humains. Terrence Deacon (né en 1950) avance l'idée selon laquelle le langage (et l'ensemble de notre monde symbolique) dérive d'une auto-domestication de l'humain par l'humain. Dans deux ouvrages majeurs (The Symbolic Species en 1997, et Incomplete Nature en 2011), et d'innombrables articles, Deacon a développé une hypothèse complexe et transversale, qui emprunte à l'anthropologie et aux neurosciences (que le scientifique enseigne à Berkeley), comme à la sémiotique et à la biologie, en vue d'expliquer l'émergence des propriétés de l'esprit humain jugées les plus singulières. Dans cette perspective naturaliste, l'énigme du singe parlant se résorbe par la co-évolution de l'anatomique et du symbolique.

L. D.

Texte original publié le 14 février 2010 sur le forum en ligne « On the Human » du National Humanities Center ${ }^{1}$. Le format a été revu pour convertir les références bibliographiques citées en note de bas de page.

\section{Introduction}

Il arrive qu'une complexité extrême mette le modèle de la sélection naturelle au défi d'expliquer quoi que ce soit. Depuis Darwin, l'aptitude humaine au langage est incessamment citée en exemple-type de ce cas de figure. Et ceux qui ont souligné les problèmes posés par cette faculté si spécifiquement humaine n'étaient pas tous des critiques du darwinisme. On sait l'argument avancé par Alfred Russel Wallace, co-instigateur de la théorie de la sélection naturelle, et réputé plus darwiniste que Darwin à bien des égards: selon lui, en devenant apte au langage, l'intellect humain

1. http://onthehuman.org/2010/02/on-the-human-rethinking-the-natural-selection-of-human-language/ (visitée le 23 août 2011). Noter que cette page comprend également une annexe de commentaires nourris sur le texte, et une longue réponse de l'auteur à leur suite. 
a franchi un niveau de complexité excédant de beaucoup celui que la sélection naturelle seule aurait pu atteindre. Farouchement attaché à cette dernière pour expliquer les traits typiques d'autres espèces, Wallace n'en souligna pas moins que « la sélection naturelle aurait tout juste permis au cerveau de l'homme primitif de surpasser celui du singe ${ }^{2} »$. Quant à Charles Lyell, qui s'employa personnellement à faire connaître les travaux de Darwin et se prononça, d'une manière générale, en faveur de la théorie évolutionniste, il s'inquiéta lui aussi de ce que le langage fût manifestement trop complexe pour ressortir à une évolution naturelle 3 . Même les plus simples des langues naturelles ont un vocabulaire d'une ampleur, une structure grammaticale et syntaxique d'un baroque, qui dépassent en complexité les systèmes de communication utilisés par les autres espèces quelles qu'elles soient. Et de plus ces langues permettent d'exprimer des concepts ésotériques ou de transmettre des expériences esthétiques, résultat qu' aucun processus directement adaptatif ne semble en mesure d'égaler.

Darwin lui-même envisageait avec tracas que la sélection naturelle ne pût rendre compte de phénomènes naturels fonctionnellement très complexes. Dans une lettre à Asa Gray immédiatement postérieure à la publication de l'Origine des espèces, il reconnaît que « la vue d'une plume de paon me rend instantanément malade 4 ! ». Le fait est qu'un tel ornement, nonobstant la spectaculaire richesse et la minutieuse finesse de ses détails, ne pouvait avoir d'effets que négatifs sur la santé et la survie de l'espèce; il n'aurait par conséquent pas dû être retenu par une sélection naturelle corrélée à l'environnement. Mais précisément ce sont de semblables extravagances qui, en dépit de leur inutilité, ont inspiré à Darwin une manière d'approcher la redoutable question des facultés mentales humaines.

Pour la plume du paon comme pour d'autres traits similaires, Darwin comprend que la sélection naturelle conditionnée par l'environnement n'a pas joué un rôle déterminant. La reproduction est, reconnaît-il, un facteur d'évolution autrement crucial que la survie individuelle: c'est

2. Alfred Russel Wallace, «Sir Charles Lyell on Geological Climates and the Origin of Species », Quarterly Review, CxxvI (1869), p. 359-394, ici p. 392.

3. Voir Charles Lyell, Geological Evidences of the Antiquity of Man, Londres, John Murray, 1863.

4. Lettre à Asa Gray, 3 avril 1860: Darwin Correspondence Database, lettre 2743, URL: www. darwinproject.ac.uk/entry-2743 (visité le 23 août 2011).Cf. On the Origin of species by means of natural selection, or the preservation of favoured races in the struggle for life, Londres, John Murray, 1859. 
pourquoi la lutte pour l'accès aux fonctions reproductrices (ou sélection sexuelle) peut provoquer une sélection débridée de certains traits, quelle que soit leur (in)adaptation au milieu. Le caractère spectaculaire ou la combativité, en ce qu'ils assurent un accès privilégié aux partenaires d'accouplement, peuvent ainsi selon Darwin voir leur prolifération et leur exacerbation favorisées au cours de l'évolution, fût-ce au détriment de la santé ou de la survie de chaque individu. Or pourquoi cette sélection sous condition de sexualité ne permettrait-elle pas, par analogie, d'expliquer des caractères aussi extravagants et déviants que ceux du langage humain? C'est ce que postule Darwin dans La Filiation de l'homme et la sélection liée au sexe - livre dont on cite habituellement le titre en l'amputant de sa seconde moitié 5 . Il y propose d'expliquer le langage, et d'autres traits dont le développement semble hors de proportion avec leur utilité pour la survie, en tant qu'ils résulteraient de la sélection sexuelle. Ainsi imagine-t-il que le langage ait pu être à l'origine quelque chose comme un chant d'oiseau, utilisé pour attirer les partenaires, et qu'au fil du temps la faculté d'émettre des comportements vocaux hautement élaborés se soit développée de manière disproportionnée, sous l'effet d'une course à l'échalote visant à faire étalage du plus de complexité vocale possible.

S'agissant d'une faculté aussi spécifiquement humaine que le langage, on ne peut malheureusement que nourrir les plus sérieux doutes à l'égard de l'idée de sélection sexuelle. Celle-ci en effet provoque inévitablement l'émergence de caractères mâles et femelles complémentairement divergents, ainsi qu'en témoignent la plume du paon ou la ramure de l'élan, que seuls les mâles exhibent. Or si hommes et femmes présentent effectivement quelques signes distinctifs caractérisés (comme le mode de répartition des tissus adipeux sur la poitrine ou les hanches, etc.), en termes d'aptitudes intellectuelles et langagières la différence des sexes reste des plus discrètes. Rendre compte de l'extravagante complexité du langage par la sélection sexuelle suppose ainsi d'expliquer pourquoi, là où partout règne un dimorphisme sexuel extrême, ici ne s'en repère aucune trace. C'est toute la difficulté que relevait Wallace: nous avons affaire à un mode d'adaptation éminemment structuré, unique à l'humain,

5. Charles Darwin, The Descent of man and selection in relation to sex, Londres, John Murray, 1871. [Trad. fr.: La Descendance de l'homme et la sélection sexuelle (par Jean-Jacques Moulinier), Paris, C. Reinwald, 1872 ; nouvelle trad. La Filiation de l'homme et la sélection liée au sexe (coordonnée par Michel Prum), Paris, Syllepse/Institut Charles Darwin International, 1999 (N.d.T.).] 
mais dont les caractéristiques très complexes ne semblent pas pouvoir s'expliquer comme résultant d'une adaptation à strictement parler.

\section{L'évolution en niche artificielle sur le long terme}

Dans mes travaux j'utilise l'expression espèce symbolique, entendue à la lettre, pour faire valoir que nous avons été, en tant qu'organismes biologiques, littéralement transformés par les symboles ${ }^{6}$. À mon sens les bizarreries dont témoignent nos pensées et nos comportements par comparaison avec les autres espèces sont imputables aux modifications que le langage a opérées en nous. À bien des titres le langage symbolique a été un élément essentiel de l'environnement auquel nous avons dû nous adapter pour proliférer. C'est dans le contexte des exigences imposées par la recherche de nourriture en station debout, avec l'aide d'outils lithiques, et par le développement d'un régime alimentaire carné, que les corps de nos ancêtres évoluèrent. De même c'est dans le contexte d'une communication richement tissée de culture symbolique que leurs cerveaux se transformèrent. Pour survivre et se reproduire, il devint de plus en plus essentiel de pouvoir s'insérer dans le réseau social des premières formes (protolinguistiques ou autres) de communication symbolique: ainsi les facultés mentales garantissant l'accès à cette ressource vitale durent se trouver mises en avant de manière sélective, sous l'effet des contraintes imposées par cette niche artificielle. Nous ne sommes donc pas simplement des créatures dotées d'intelligence ou de sagesse (sapiens): nos facultés sociales et mentales ont été littéralement formées par les nécessités de la communication symbolique. Cela a impliqué des adaptations non seulement pour l'emploi du langage, mais aussi pour tous les auxiliaires mentaux contribuant à assurer l'obtention et l'usage de cette ressource sociale.

Cette proposition implique néanmoins que la communication de type langagier ait été de longue date un trait de l'évolution hominidée. Les théories selon lesquelles le langage est un phénomène évolutif aussi soudain que récent ne feront pas la même hypothèse. Leur conception du langage est quasiment épiphénoménale. Certains en particulier supposent qu'il a surgi tout à coup, comme par enchantement: prodigieuse mutation qui

6. Voir Terrence W. Deacon, The Symbolic Species : the coevolution of language and the brain, New York, W. W. Norton \& Co., 1997. 
transforma accidentellement des brutes épaisses (mais à volume cérébral important) en beaux parleurs. Les éléments invoqués pour corroborer ce type de scénario sont des indices très indirects pour la plupart (comme par exemple la mise au jour par les archéologues de certaines formes représentées ou d'objets ornementaux remontant au paléolithique supérieur), ce qui ne l'a pas empêché d'être communément accrédité ces dernières années. À mes yeux cela reflète principalement une vision caricaturale de la distinction humain/animal, ainsi qu'une sorte de métaphorisation héroïque forcée des fossiles exhumés. Or la façon qu'ont les cerveaux humains modernes de faire place au langage est un indice pour dater l'émergence de ce dernier.

À supposer que dans les interactions sociales humaines le langage constitue un élément comparativement récent - disons, vieux de quelque cent mille ans -, il faudrait s'attendre à ce que ses effets sur le cerveau soient demeurés limités. Quels qu'aient été les ajustements structuraux nécessaires à l'évolution de l'architecture cérébrale, de deux choses l'une : soit ils furent réduits au minimum, soit ils furent majeurs mais sans nécessiter d'importantes transformations génétiques. S'il est d'origine récente, le langage n'aura donc pas été à même d'imprimer sa marque sélective au cerveau humain; aucune modification neurologique généralisée et intégrée ne se sera produite à l'appui de la fonction langagière. On en déduit que les facultés de langage durent tant bien que mal se ménager une place au sein d'un cerveau par ailleurs typiquement simiesque, quoique plus volumineux. Elles sont principalement un après-coup de l'évolution. Car eu égard au temps imparti, la fixation génétique de caractères propices en grand nombre n'a pu se produire, l'adaptation n'a dû être assurée que par une poignée d'altérations génétiques et neurologiques essentielles. À cette enseigne la fonction langagière ne pourra être qu'imparfaitement intégrée aux autres fonctions cognitives, fragilisée par la moindre carence d'apprentissage, soumise à de catastrophiques sautes d'activité par certaines déficiences minimes mais critiques, et sévèrement affectée par tout handicap mental congénital.

Rien de tout cela ne semble devoir se vérifier.

À l'inverse, si le langage est parmi nous depuis une bonne partie de notre évolution - disons, un bon million d'années - cela aura suffi à ce que les exigences du langage se fassent connaître au cerveau avec plus d'ampleur. Un vaste filet de subtiles altérations génétiques et d'ajustements neurologiques aura été lancé, avec pour résultat la constitution d'une 
fonction neurologique remarquablement robuste et intégrée. De fait, nombreux sont les indices attestant que le langage a intégré la quasi-totalité de notre vie cognitive et sociale, qu'il mobilise une part significative du cerveau antérieur, et qu'une fois acquis il résiste aux circonstances sociales et aux infirmités neurologiques les plus lourdes. Le langage est tout sauf fragile.

La co-évolution interactive joue en fait dans les deux sens. Les langues, elles aussi, ont dû s'adapter aux cerveaux. Chaque langue devant, une fois apprise, être transmise à la génération suivante, il lui faut pour se reproduire efficacement des structures faciles à assimiler, adaptées aux limites de l'humain. Langues et cerveaux évoluent donc en tandem, leurs trajectoires convergeant - quoique de manière asymétrique - l'une vers l'autre. Sans doute l'évolution cérébrale avance-t-elle à pas comptés, tandis que le procès langagier est plus alerte: il faut s'attendre à ce que celui-ci s'adapte davantage à celle-là que l'inverse. Pour autant, s'il y a déjà un million d'années que nous évoluons ainsi en niche symbolique, le cerveau humain doit bien avoir conçu différents trucs et astuces pour simplifier la vie dans ce monde virtuel.

L'univers des symboles forme une niche artificielle. Son écologie diffère foncièrement de celle de la niche biologique où nous nous trouvons également - ou du moins nos ancêtres se trouvaient. De même qu'en construisant des barrages les castors ont créé une niche aquatique à laquelle leur corps s'est adapté au cours de son évolution, de même nos facultés cognitives ont-elles été infléchies par la niche que nous nous sommes construite: niche symbolique, celle-là. L'idée n'est d'ailleurs pas nouvelle: l'anthropologue Clifford Geertz formula un argument approchant il y a plusieurs décennies de cela. Mais il me semble être aujourd'hui temps que nous commencions à en étudier de près les implications, au point où nous en sommes de notre théorisation de l'évolution et de notre compréhension du cerveau.

Au prisme de la divergence entre la cognition humaine et les modalités plus typiques d'autres espèces, il doit être possible d'examiner les contraintes propres à la niche symbolique dans ce qu'elles ont de plus intense et de plus inhabituel. Il est depuis longtemps convenu de penser la différence humaine en termes d'intelligence en général : à mon sens c'est se fourvoyer, car ce faisant on ignore une constellation plus essentielle, formée de différences plus subtiles. Certaines parmi cellesci pourraient concerner la cognition sociale (s'agissant par exemple de l'attention conjointe, de l'empathie, de l'aptitude à anticiper les actions 
intentionnelles d'autrui), nos manières d'apprendre (ainsi une aptitude supérieure à l'apprentissage par transferts d'informations, une tendance à supposer que les associations sont bidirectionnelles - dite aussi équivalence des stimuli - la facilité relative dans l'imitation), ou même simplement des facultés motrices inhabituelles (comme un contrôle articulatoire et vocal sans précédent). Toutes forment un vaste et divers ensemble d'adaptations qui, en bloc ou au détail, font parties intégrantes de nos facultés langagières.

Quant au cerveau, nous sommes face à une énigme supplémentaire: comment l'immense diversité de ses traits a-t-elle pu donner lieu à un entrelacs interdépendant si fonctionnellement propice au nouveau mode de communication? La question a ceci d'épineux que le langage est un phénomène émergent, non pas une fonction a priori requérant de simples ajustements. À l'instar des autres modes de communication utilisés par les animaux, les diverses vocalisations dont nous avons hérité, comme le rire, le cri d'effroi ou les pleurs d'angoisse, mobilisent des zones relativement localisées du cerveau, principalement dans les régions sous-corticales. Par comparaison, le langage mobilise une constellation vaste et dispersée de systèmes corticaux, qui se retrouvent dans le cerveau d'autres primates mais y ont évolué pour satisfaire à des fonctions bien différentes. Comment ces systèmes cérébraux se sont-ils trouvés collectivement enrôlés pour servir au langage, bien que leur évolution les ait disposés pour un tout autre office? C'est uniquement parce que les fonctions vers lesquelles ils avaient précédemment évolué recoupaient largement certaines des opérations requises pour la production et la compréhension du langage. Celles-ci relèvent en effet de structures et de circuits neuronaux homologues à ceux que l'on trouve omniprésents dans la plupart des cerveaux simiesques. Ainsi une configuration ancienne fit l'affaire pour s'acquitter d'une tâche pourtant sans précédent.

Une autre question encore se joint à la précédente: dans quelle mesure cette forme prédominante de communication qu'est le langage dépend-elle de signaux entretenus et transmis socialement? Même dans le cas où l'on postule une grammaire universelle innée, le vocabulaire standard procure déjà assez de richesse et de finesse d'information pour ne pas apparaître extrêmement anormal d'un point de vue biologique. Comment une aussi importante partie de notre faculté à communiquer se trouva-t-elle au bout du compte confiée aux bons soins d'une transmission sociale ? Et à quoi ce processus doit-il sa remarquable fiabilité? 


\section{Sélection relâchée et complexité}

La plus inattendue, et la plus discutable peut-être, des propositions avancées ici, tient à l'importance que j'accorde à la sélection relâchée ${ }^{7}$. La synergie systémique d'ordre supérieur qui prend part au langage requiert des systèmes constituants du cerveau qu'ils adoptent un fonctionnement coopératif. Mais celui-ci suppose, paradoxalement, que la synergie des différents systèmes ait été mise en place au préalable, afin que la sélection en assure l'affûtage pour le compte du langage.

Pour expliquer comment s'est construite la niche symbolique, j'ai esquissé précédemment un scénario de co-évolution. Mais dans celui-ci, l'engendrement d'une nouvelle synergie fonctionnelle des systèmes neuronaux, telle que la requiert l'opération langagière, demeure inexpliqué. Nous sommes en présence de deux systèmes cérébraux de contrôle, l'un du cri, l'autre de la parole et du langage: entre les deux, les solutions de continuité rendent impossible d'invoquer une simple logique de co-évolution afin d'expliquer la modification du substrat. Les recherches récemment entreprises à propos du chant des oiseaux, qui mettent en évidence des transformations survenant concomitamment en matière de complexité et de substrat neuronal, pourraient nous aider à y voir plus clair.

L'étude porte sur la comparaison d'un oiseau depuis longtemps connu sous forme domestique, le capucin bistré, avec son cousin sauvage, le capucin domino ${ }^{8}$. Elle a établi que l'oiseau d'élevage acquiert beaucoup plus facilement le chant, et est capable d'une mélodie nettement plus complexe et flexible que ses cousins à l'état sauvage. Et ce, bien que le capucin bistré ait été élevé en captivité pour la couleur de son plumage, non pour celle de son ramage ${ }^{9}$. La différence entre ces deux très proches types de capucin, l'un sauvage, l'autre domestique, dans l'apprentissage et la complexité du chant, recoupe celle qu'ont mise en évidence des comparaisons entre les espèces capables d'apprendre à chanter et celles qui ne le sont pas. Elle correspond aussi à la présence, chez les oiseaux

7. Cf. Terrence W. Deacon, « Relaxed selection and the role of epigenesis in the evolution of language », dans M. S. Blumberg, J. H. Freeman, S. R. Robinson (dir.), Oxford Handbook of Developmental Behavioral Neuroscience, New York/Oxford, Oxford University Press, 2009, p. 730-752.

8. Respectivement Lonchura striata domestica (Linnæus, 1758) et Lonchura striata (Linnæus, 1766). (N.d.T.)

9. Kazuo Okanoya, « The Bengalese Finch: a window on the behavioral neurobiology of birdsong syntax », Annals of the New York Academy of Science 1016 (2004), p. 724-735. 


\section{Comment repenser la sélection naturelle du langage humain}

capables d'acquérir des mélodies complexes et variables, d'une bien plus complète maîtrise neuronale du chant.

Il est surprenant que cette complexité comportementale et neuronale se développe de manière spontanée, sans faire l'objet d'un élevage spécifique, car généralement la complexification du chant est supposée se produire sous la pression d'une intense sélection sexuelle. Or la domestication coupe court à celle-ci. D'où l'hypothèse engageante suivant laquelle, dans le présent cas de figure, c'est le relâchement de la sélection naturelle et sexuelle exercée sur le chant qui, paradoxalement, serait la cause de sa complexification. En bref, dès lors que le chant n'est plus pertinent pour identifier l'espèce, défendre le territoire, attirer les partenaires et éviter les prédateurs, rien n'évince plus les mutants détériorés et les allèles nuisibles susceptibles d'affecter la fixation d'un chant stéréotypé. Il en résulte un amenuisement des biais innés qui bordaient la production du chant, c'est-à-dire une recrudescence des types de perturbation subies par celle-ci. À l'état domestique, le chant semblera ainsi sujet à moins de restrictions et plus de variations. Mais comme la détermination de sa structure n'est plus désormais étroitement contrôlée par la région motrice du cerveau antérieur primaire (appelée noyau RA), d'autres systèmes cérébraux peuvent alors s'en mêler. Les biais moteurs innés étant atténués, l'expérience auditive, le contexte social, les préférences d'apprentissage et les facteurs d'attention se mettent à influer sur le chant. Résultat: l'oiseau domestique varie et complexifie les effets, il se laisse davantage influencer par son expérience sociale. Le recombinaison aléatoire des allèles accroissant la diversité génétique et phénotypique au sein d'une population, la sélection relâchée provoque habituellement un effet de dérive génétique; neurologiquement parlant, néanmoins, le relâchement du contrôle génétique exercé sur les fonctions neuronales devrait se solder par une dilution des contraintes, d'où un accroissement de la flexibilité comportementale et de la sensibilité conditionnelle à d'autres facteurs neuronaux et contextuels.

Tout cela a-t-il un rapport avec l'homme? Oui, puisque le processus adaptatif du langage humain relève par de nombreux traits d'un relâchement des contraintes innées, et qu'ainsi la vocalisation a évolué sous des influences plus multiples que les réactions-types aux émotions et au contexte immédiat. L'indice le plus net en est probablement le babillage enfantin. Cette tendance sans précédent au libre jeu de la voix ne fait guère intervenir de contrainte innée quant aux séquences sonores possibles (si 
ce n'est celle qu'exerce la production physique du son vocal). De plus, le babillage survient en contexte de faible excitation, là où les rires, les pleurs et les cris relèvent de stimulations relativement intenses et associées à des contextes spécifiques. Moins soumise aux contraintes innées d'excitation et de contexte, la production du son peut dès lors se prêter à de nombreuses autres influences. Comme pour l'oiseau domestiqué, le comportement vocal laisse intervenir une grande diversité de systèmes cérébraux, sans oublier l'expérience auditive socialement acquise. Au vrai, ce desserrement de la contrainte est un préalable essentiel à ce que des comportements vocaux acquis puissent être mis en correspondance avec la multitude d'objets, d'événements, de propriétés et de relations que le langage est à même de dénoter. Il constitue également une réponse plausible à la question de la synergie combinatoire que nous nous posions plus haut: en effet, le mécanisme évolutif mis en évidence ici aura eu spontanément pour effet la maîtrise neuronale du comportement vocal par la coordination de plusieurs systèmes entre eux.

Un tel processus évolutif de dédifférenciation contribue à expliquer les adaptations du langage humain. Nous ne saurions cependant en rester là. Il est probable que, grâce à la multiplication de la flexibilité et de la conditionnalité, des interrelations auparavant incongrues entre systèmes cérébraux aient été sélectionnées pour servir aux nouvelles associations fonctionnelles qui avaient émergé : autant d'adaptations dont l'identification reste à faire. Mais quoi qu'il en soit, si tel est l'effet de dédifférenciation dont relève notre évolution, les hypothèses faisant fond sur une sélection par l'inné, ou sur une extrapolation d'après l'inné, voient la plausibilité de leurs scénarios réduite d'autant.

\section{Quelques spéculations pour conclure}

Je souhaiterais pour finir aborder certains traits de l'humain parmi les plus ésotériques, en supposant qu'ils puissent être mieux compris à la lumière des deux processus étudiés ici: la construction d'une niche symbolique et la sélection relâchée.

Il me semble précieux de nous penser nous-mêmes en tant que porteurs d'un savoir symbolique [symbolic savants], incapables de réprimer toutes les prédispositions dont l'évolution nous a dotés pour favoriser l'acquisition, l'usage et la transmission des symboles. Pour être à ce 
point rompus à cette étrange tâche cognitive, nous devons certainement avoir développé une propension à considérer chaque chose comme un symbole, qu'elle le soit ou non. À preuve le faire-semblant des enfants, la signification que nous prêtons à de simples coïncidences, les visages que nous distinguons dans les nuages, l'art qui nous envoûte, la musique qui nous enchante, nos vies présumées obéir aux voix d'esprits invisibles. Les oiseaux jouent en vol, les singes manipulent des objets, les chats raffolent de joujoux à plumes : de même les spécificités de notre adaptation réfractent notre vision du monde. Ainsi sommes-nous irrépressiblement enclins à rechercher le sens cryptique que dissimulent les apparences. Nul doute que bon nombre de nos aptitudes et préférences sociales propres - tendance au conformisme, souci de l'enfant de reproduire les paroles entendues - reflètent aussi ces adaptations à un écosystème de relations symboliques. Sans parler de la littérature et du théâtre. Voyez comme nous pouvons sans effort nous mettre à la place d'autrui, sentir ses joies et ses peines presque aussi intensément que si elles étaient les nôtres.

Le relâchement de la sélection, de son côté, pourrait avoir contribué à définir tout un ensemble de caractères spécifiquement humains. Une dédifférenciation génétique et épigénétique de grande ampleur aura eu pour effet de rendre plus flexibles divers systèmes de cognition et de motivation, auparavant phylogénétiquement déterminés. Parmi les traits caractéristiques des humains, le plus saillant pourrait être leur flexibilité et leur diversité culturelle. Celle-ci, s'agissant par exemple de mariage et de parenté, confine à l'incroyable. Pour la plupart des espèces l'association conjugale et familiale, comme les soins dont la descendance est entourée, répondent à des schémas pour le moins prévisibles, et bien que flexibles ils varient uniquement en fonction de systèmes de motivation individuelle. Chez l'homme, par contraste, et en dépit de l'importance de la reproduction dans son évolution, les liens conjugaux et parentaux dépendent pour l'essentiel de négociations sociales à visée symbolique. Ainsi des fonctions parmi les plus fondamentales du monde biologique se trouvent-elles confiées à des mécanismes socio-symboliques : là réside, peut-être, la marque de fabrique d'une espèce symbolique. De la sorte, du fait des symboles et grâce à eux, Homo sapiens s'est domestiqué luimême en se coulant dans une niche sans équivalent connu. Le mot nous a conçus à son image.

Traduit de l'anglais (États-Unis) par Marc Aymes. 